\title{
Potential New Paradigm for Treatment of Atopic Dermatitis
}

Paller AS, Eichenfield LF, Kirsner RS, et al.; US Tacrolimus Ointment Study Group: Three times weekly tacrolimus ointment reduces relapse in stabilized atopic dermatitis: a new paradigm for use. Pediatrics 2008, 122:e1210-e1218.

Rating: $\bullet$ Of importance.

Introduction: Atopic dermatitis is the most common chronic inflammatory skin disease in children. As topical corticosteroids have local and system side effects, safe and effective treatments that maintain longer remissions are needed. This trial examined the use of three-times-weekly tacrolimus ointment in maintaining remission.

Aims: The primary aim of this study was to determine whether the long-term intermittent application of a topical calcineurin inhibitor (TCI) was safe and efficacious.

Methods: This was a multicenter, two-phase study of 206 patients 2 to 15 years of age with moderate to severe atopic dermatitis. In the first phase of the study, the goal was to stabilize patient symptoms. The stabilization phase was divided into two periods. The first period was double-blinded, and patients were assigned to use tacrolimus ointment, $0.03 \%$, or alclometasone ointment, $0.05 \%$, for 4 days. During the second period, all patients received tacrolimus ointment, $0.03 \%$ (open-label), for up to 16 weeks. The maintenance phase was a randomized, double-blind, placebo-controlled evaluation of tacrolimus ointment, $0.03 \%$, or vehicle ointment applied once daily or three times weekly to the previously affected sites.

Results: There was no difference in adverse events between alclometasone and tacrolimus or between tacrolimus and placebo. In addition, early treatment with alclometasone was superior to tacrolimus. Finally, the tacrolimus-treated patients had significantly more disease-free days, longer time to first relapse, and fewer disease-relapse days compared with the vehicle-treated group.

Discussion: After initially stabilizing disease with topical corticosteroids, long-term, intermittent application of a TCI appears to be a safe and efficacious strategy to maintain remission of moderate to severe atopic dermatitis in children 2 years of age and older.

\section{Comments}

This article provides an intriguing new paradigm for use of TCI in atopic dermatitis. Unfortunately, these medications continue to carry a black-box warning noting that the long-term safety of TCI has not been established and that rare cases of cancer have been reported in patients who used the medications, although a causal relationship has not been established. Although controversial, this warning has prompted many practitioners to avoid this class of medications until further information regarding long-term safety compared with that of topical corticosteroids is established. Considering their potential benefits, perhaps it is time to take a fresh look at TCIs.

\section{Acknowledgment}

Drs. Christina E. Ciaccio and Jay M. Portnoy are affiliated with the Section of Allergy, Asthma, and Immunology at the Children's Mercy Hospitals and Clinics, where Dr. Ciaccio is an Assistant Professor of Pediatrics and Dr. Portnoy is the Chief of the Section of Allergy, Asthma, and Immunology and a Professor of Pediatrics. Correspondence should be sent to 2401 Gillham Road, Kansas City, MO 64108, USA. E-mail: ceciaccio@cmh.edu; jportnoy@cmh.edu.

\section{Disclosure}

No potential conflicts of interest relevant to this article were reported. 